\title{
Cranium bifidum occulta: case report and review of the differential diagnosis
}

\author{
Farzad Tahmasebi Arashloo'; Mohsen Shahba²; Farnaz Fahimi Hanzaei ${ }^{1}$; Ghazaleh Amjad ${ }^{3 *}$ \\ ${ }^{1}$ Department of Radiology, Kerman University of Medical Sciences, Iran \\ ${ }^{2}$ Department of Neurosurgery, Kerman University of Medical Sciences, Iran \\ ${ }^{3}$ Shahid Akbar Abadi Clinical Research Development Unit (ShACRDU), Iran University of Medical Sciences (IUMS), Tehran, Iran
}

\section{*Corresponding Author(s): Ghazaleh Amjad}

Shahid Akbar Abadi Clinical Research Development Unit (ShACRDU), Iran University of Medical Sciences (IUMS), Tehran, Iran

Tel: 00-989126206820, Fax: 00-982188374879, Email: ghazal_1986_amjad@yahoo.com

Received: Nov 24, 2018

Accepted: Dec 21, 2018

Published Online: Dec 28, 2018

Journal: Journal of Case Reports and Medical Images

Publisher: MedDocs Publishers LLC

Online edition: http://meddocsonline.org/

Copyright: (C) Amjad G (2018). This Article is distributed under the terms of Creative Commons Attribution 4.0 International License

\section{Case report}

A full term female neonate who was born after a cesarean section with no history of head trauma or difficult birth was evaluated for a skull deformity and large cranial defect. The mom did not have any complications during pregnancy. Mother also denied any history of alcohol consumption and smoking, there was no history of infection during her pregnancy. The first child of the family was a 10-year-old girl with normal appearance and normal growth during her childhood. The newborn's APGAR score was 10. At birth, the neonate's weight was $3150 \mathrm{gr}$ and her length was $53 \mathrm{~cm}$ and her head circumference was 37 $\mathrm{cm}$. In general examination, the newborn had dysmorphic face with scalp depression in bilateral parietal regions. The superior portion of both frontal, parietal and occipital bones showed un-

\begin{abstract}
Bilateral hypoplasia of parietal bones is a rare clinical finding. In this article we introduced a neonate with chief complaint of skull deformity with no history of difficult birth or head trauma. The patient was presented for skull depression mostly in bilateral parietal regions.
\end{abstract}

der development. The metopic, coronal, and lambdoid sutures were separated and sagittal suture didn't exist because of nonproduction of both bony parietals. The anterior and posterior fontanels were abnormally wide. In parietal regions, small bony lesions in inferior portions were palpable and a thin layer of skin covered most of the brain tissue (Figure1). The rest of physical exam was unremarkable.

In laboratory tests, patient had normal serum calcium, phosphorus, and alkaline phosphatase (ALP) levels along with normal liver and renal function tests. Levels of thyroxine, thyroid stimulating hormone, parathyroid hormone, and $25-\mathrm{OH}$ vit $\mathrm{D}$ levels were inconspicuous.

Cite this article: Arashloo FT, Shahba M, Hanzaei FF, Amjad G. Cranium bifidum occulta: Case report and review of the differential diagnosis. J Case Rep Clin Images.2018; 3: 1012. 


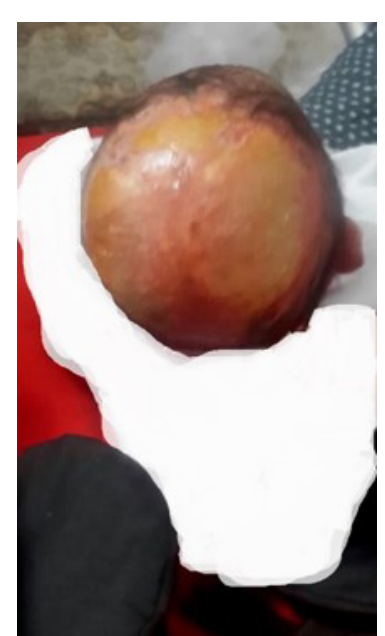

Figure 1: Thin layer of skin covers the brain tissue.

Bilateral hypoplasia of parietal, occipital and frontal bones associated with widening of all sutures mostly in the sagittal suture were determined in Computed Tomography (Figure 2). Brain parenchyma and extra-axial space were normal and no obvious soft tissue mass or vascular lesions or ventriculomegaly were detected.

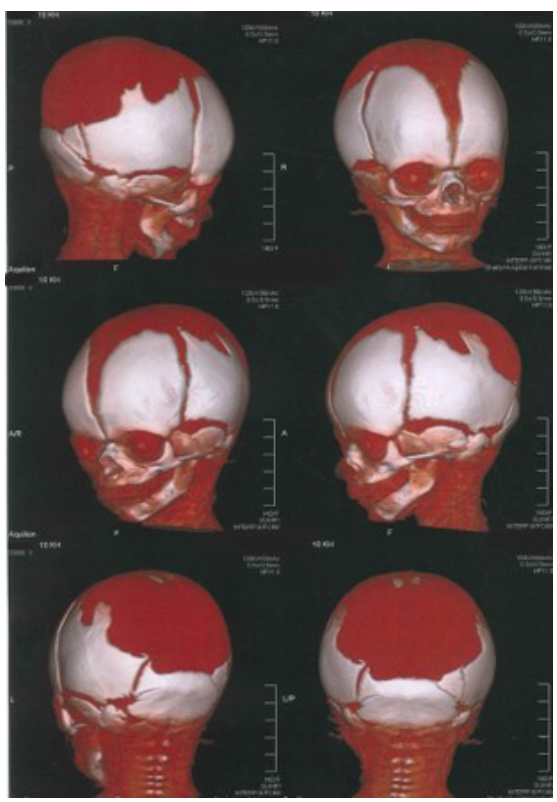

Figure 2: Three- dimensional reformats CT scan, showing the extent of the bony defect soon after birth.

In obstetric sonography, cranium had low echogenicity and the fetal head was compressible by the ultrasound probe. Also, brain parenchyma and both ventricles were clearly visible. These findings led to suspicion of skeletal dysplasia such as osteogenesis imperfecta or achondrogenesis and hypophosphatasia. But, normal alignment and length of long bones, ribs, and axial skeleton in addition with negative family history reduced the possibility of dysplasia.

So we diagnosed the patient's disease as cranium Bifidum occulta.

\section{Discussion}

Congenital calvarial defects are a group of disorders that vary in their severity. Cranium bifidum occultum (cleft skull) is a rare condition which is due to delayed ossification of parietal bones with subsequent large midline skull defect. In true cranium bi- fidum, the herniation of meninges or brain parenchyma may be perceived. Defect in formation of the bone or failure of primary neural tube closure could be the etiology of this disease [1].

There are several possible differential diagnoses. The diagnosis should be made by excluding the other disorders.

Generalized decrease in skull density and severe thinning of the calvaria in the neonate may be seen in some skeletal dysplasia such as osteogenesis imperfecta, hypophosphatasia, achondrogenesis, or Menkes syndrome. But in these disorders, presence of other skeletal anomalies or laboratory impairment is indispensable. For instant, in hypophosphatasia which is due to absent or low activity of serum alkaline phosphatase, the ossification of skull and vertebrae is reduced and the tubular bones are short with frayed metaphyses similar to rickets. Menkes syndrome which is a disorder of copper metabolism manifests with osteopenia, mental retardation, micrognathia, metaphyseal spurs, and urinary tract anomalies. The serum copper level is also elevated [2].

The differential diagnosis for focal osteopenia in the newborn skull is too restricted. Localized reduction in ossification can be appeared in the lacunar skull. The lacunar skull is a disorder of the membranous bone that is related to non-ossified fibrous bone and shows well-defined lucent areas in the calvaria which is encircled by normally ossified bone. This is not related to the degree of hydrocephalus and disappears by age of 6 months. Neural tube defects particularly myelomeningocele with chiari II malformation or encephalocele are seen in companion with lacunar skull [3].

Pycnodysostosis, cleidocranial dysostsis, and congenital hypothyroidism could be recognized as differential diagnosis due to their open fontanels and cranial sutures.

In cleidocranial dysostosis, the involvement of the clavicle, multiple wormian bones, frontal and parietal bossing, basilar invagination, supernumerary teeth, abnormal ear structures, hypoplasia of iliac bones and delayed ossification of pubic bone with pseudo widening appearance of the symphysis pubis are other accompanying findings [4].

In pycnodysostosis, major clinical manifestation includes short stature, large head with frontal and parietal bossing, obtuse mandibular angle, dental abnormalities, short hands, and feet with dystrophic nails. In radiologic survey, increased bone density, open anterior fontanels and sutures, non-pneumatized paranasal sinuses, flattened mandibular angle, aplasia of acromial end of clavicle and acroosteolysis are the main imaging findings [5].

Bilateral thinning of the parietal bones is a condition which contemplated as a non-progressive congenital dysplasia of the diploe or as an acquired disease. The area between the parietal prominence and the sagittal suture is the most prevalent site. Symmetrical thinning of bilateral parietal bones involving the diploe and the outer table with intact contour of inner table are demonstrated in radiographic examinations [6].

Although the parietal bone defects are rare, the most congenital defects include parietal foramina which is a consequence of incomplete or delayed ossification of this bone. It can be seen mostly as an isolated phenomena or as part of syndrome. These foramina may stay until adult life but after few month of life, ossification of midline part will disperse the confluent defect [7]. 
The treatment of occult cranium bifidum is frequently wait and watch due to its benign nature. Surgery is demanded for persistent cranium bifidum [8].

\section{Conclusion}

Isolated hypoplasia of cranial vault bones mostly seen in parietal bones and is a rare clinical finding. The diagnosis should be discriminated by excluding the other skeletal dysplasia or metabolic diseases.

\section{References}

1. Barkovitch AJ. Pediatric neuroimaging. 2nd ed. New York, NY: Raven. 1995.

2. Williams PL. Embryology and development. In: Bannister $\mathrm{LH}$, Berry MM, Collins P, Dyson M, Dussek JE, Ferguson MW, eds. Gray's anatomy: the anatomical basis of medicine and surgery. 38th ed. New York, NY: Churchill Livingstone. 1995; 271-275.

3. Naidich TP, Pudlowski RM, Naidich JB, Gornish M, Rodriguez FJ. Computed tomographic signs of the Chiari II malformation. Part I: skull and dural partitions. Radiology. 1980; 134: 65-71.

4. Fratzl-Zelman N, Valenta A, Roschger P, Nader A, Gelb BD, et al. Decreased bone turnover and deterioration of bone structure in two cases of pycnodysostosis. J Clin Endocrinol Metab. 2004; 89: 1538-1547.
5. Maroteaux P, Lamy M. Pyknodysostosis. Presse Med. 1962; 70: 999-1002.

6. Lim Seok Tae, Sohn Myung-Hee. Bilateral Symmetric Photon Defects in the Parietal Bones on Tc-99m MDP Bone Scintigraphy Bilateral Parietal Thinning. Clinical Nuclear Medicine. 2001; 26: 570-571.

7. Mavrogiannis LA, Antonopoulou I, Baxova A, et al. Haploinsufficiency of the human homeobox gene ALX4 causes skull ossification defects. Nat Genet. 2001; 27: 17-18.

8. Wilkie AOM, Mavo giannis LA. Enlarged parietal foramina/ Cranium bifidum. In: Pagon RA, Bird TD, Dolan TD, Stephens K, eds. Gene Reviews [Internet]. Seattle (WA): University of Washington, Seattle. 1993-2004. 